\title{
Multi-Objective Optimal Charging Control of Plug-In Hybrid Electric Vehicles in Power Distribution Systems
}

\author{
Wei Li ${ }^{1}$, Zhiyun Lin ${ }^{2, *}$, Kai Cai ${ }^{3}$, Hanyun Zhou ${ }^{4}$ and Gangfeng Yan ${ }^{1}$ \\ College of Electrical Engineering, Zhejiang University, Hangzhou 310027, China \\ 2 School of Automation, Hangzhou Dianzi University, Hangzhou 310018, China \\ 3 Department of Electrical and Information Engineering, Osaka City University, Osaka 5588585, Japan \\ 4 Institute of Marine Information Science and Technology, Zhejiang University, Zhoushan, 316022, China \\ * Correspondence: linz@hdu.edu.cn; Tel.: +86-571-8795-2152
}

Received: 30 April 2019; Accepted: 24 June 2019; Published: 3 July 2019

check for updates

\begin{abstract}
With the increasing popularity of plug-in hybrid electric vehicles (PHEVs), the coordinated charging of PHEVs has become an important issue in power distribution systems. This paper employs a multi-objective optimization model for coordinated charging of PHEVs in the system, in which the problem of valley filling and total cost minimization are both investigated under the system's technical constraints. To this end, a hierarchical optimal algorithm combining the water-filling-based algorithm with the consensus-based method is proposed to solve the constrained optimization problem. Moreover, a moving horizon approach is adopted to deal with the case where PHEVs arrive and leave randomly. We show that the proposed algorithm not only enhances the stability of the power load but also achieves the global minimization of vehicle owners charging costs, and its implementation is convenient in the multi-level power distribution system integrating the physical power grid with a heterogeneous information network. Numerical simulations are presented to show the desirable performance of the proposed algorithm.
\end{abstract}

Keywords: coordinated charging; hierarchical algorithm; multi-objective optimization; moving horizon approach; PHEVs

\section{Introduction}

Global warming caused by greenhouse gas emissions has aroused worldwide concern. In fact, the world's energy demand is currently growing sharply and the problem of the fossil fuel depletion is becoming increasingly crucial: more than 25 billion tons of $\mathrm{CO}_{2}$ arising from worldwide human activities is released annually into the atmosphere. As depicted in [1], to achieve the requirements of the European directives regarding air quality, the emission inventories are becoming more and more important in order to achieve the requirements of the European Directives regarding air quality. A collection of algorithms are proposed in [2] to estimate the emission inventory from road transport in Campania, Italy. In the emission inventory, a large portion of emitted greenhouse gas comes from the internal combustion engines of motor vehicles, significantly degrading the air quality and threatening our health. It is urgent to develop new technologies and change from conventional fuel to biofuel efficiently, both to meet the energy demands and to limit the production of carbon dioxides.

Due to the environmental benefits and high-energy efficiency, electric vehicles have been enjoying a wide prevalence from academia, the automotive industry, and governmental organizations. A plug-in hybrid-electric vehicle (PHEV), equipped with both a battery driven motor and an internal combustion engine, is more preferable as an urban green transport mode, because it is able to travel 
a long distance while reducing fuel consumption [3]. However, the increasing use of PHEVs may challenge the operation of power systems, in which the uncoordinated charging of PHEVs limits the capacity supply and aggravates the current peak load, resulting in potential security issues [4].

Fortunately, with the advancements of smart metering and communication technologies, effective algorithms focusing on controlling the timing and rate of PHEV charging have been widely studied, which is commonly called demand-side management (DSM) [5]. DSM refers to techniques that take into account the electricity consumption patterns of end-use consumers to match the current supply capacity of the power system or to forecast future supply capabilities [6]. DSM is undoubtedly an important part of the power distribution system, since it takes full advantage of the existing infrastructure to enhance the stability of the power load and achieve a low-cost for the vehicle owners in the system.

A number of studies have applied DSM to schedule the charging of PHEVs, which can fulfill a wide range of goals. A demand response strategy is proposed in [7] for coordinated charging of a group of PHEVs in parking lots, which effectively alleviates the potential new load peaks of the transformers in residential distribution networks. In [8,9], the decentralized charging control schemes are proposed to achieve valley filling, i.e., shifting the controllable PHEV load to less congested hours, while satisfying the requirements of vehicle owners. A game-based approach is proposed in [10] for coordinated charging of PHEVs. Their results show that a socially optimal equilibrium can be obtained by the proposed game and the grid optimizes its price while the vehicle owners choose their equilibrium strategies. Nevertheless, these novel schemes proposed in [7-10] focus on a single control objective for coordinated charging of PHEVs: either shifting the power load or minimizing the owners' costs. Since the load-shifting service and economic issues are not taken into account at the same time, these schemes cannot guarantee minimum fluctuation performance and economic satisfaction for a power distribution system simultaneously.

Since a power distribution system tightly integrates the energy providers with a large population of flexible loads, it is reasonable and preferable to consider multiple control objectives for the power distribution system, involving providing load-shifting services and achieving total cost minimization. In [11], based on a forecast of future electricity prices, dynamic programming is applied to achieve the optimal solution which considers an optimal charge curve and minimum expense at the same time. However, the optimal controller developed in [11] will not necessarily lead to a stable system. The authors of [12] formulate the load scheduling of PHEVs as a multi-objective constrained optimization problem with the stability of the power system considered, and a weight aggregation multi-objective particle swarm optimization (WA-MOPSO) is presented to reach the optimal solution in a smart grid scenario. However, the algorithm they propose requires each vehicle to report its local information, such as battery levels and exit times, to a central optimal controller, so its implementation requires high computation capability and may not be feasible in a large-scale network. Following the trend of distributed schemes, an augmented-constraint method is presented in [13] to achieve the optimal solution in a distributed manner, with an auxiliary service of shifting load and economic issues taken into account. Nevertheless, [13] does not consider the random arrival and departure of PHEVs, thus it is not applicable for situations where vehicle owners may arrive and leave at will.

Considering the lack of effective and practical schemes for coordinated charging of PHEVs in the power distribution system, this paper aims to solve the problem of valley filling and achieve an economically optimal solution in a distributed manner, subject to the target state-of-charge (SOC) requirements and system's technical constraints. Compared with existing works, the innovative contributions of this paper are summarized as follows:

(1) A multi-level framework of the power distribution system integrating the physical power grid with a heterogeneous information network is established for coordinated charging of PHEVs.

(2) A model of multi-objective constrained optimization with priority is employed to formulate the PHEV charging scheduling problem in the multi-level power distribution system. In detail, the objectives of enabling valley filling with a higher priority and achieving total cost minimization with a lower priority are both investigated under the system's constraints. 
(3) A hierarchical optimal algorithm combining the water-filling-based algorithm with the consensus-based method is proposed to solve the constrained optimization problem. In addition, a moving horizon method is adopted to address the random arrival and departure of PHEVs, and it is convenient to implement in the multi-level power distribution system.

The rest of this paper is organized as follows. Section 2 presents the system model, involving the multi-level framework of a power distribution system, heterogeneous information network and PHEV charging model. Problem formulation is given in Section 3. In Section 4, our main results are shown, including the primal problem transformation, hierarchical algorithm, convergence analysis, and hierarchical algorithm with moving horizon. Numerical simulations are given in Section 5. Section 6 gives the conclusion and states future work.

\section{System Model}

\subsection{Power Distribution System Modeling}

We consider a multi-level framework of a power distribution system for coordinated charging of PHEVs, as Figure 1 shows, similar to [14]. In the energy provision plane, the power grid acts as energy provider and the control centre sends control messages based on the electricity load profiles. In the energy consumption plane, there are several energy consumption areas composed of a population of PHEVs and a functional district such as a commercial building, power plant, or housing district. One area is treated as the basic unit of the energy consumption plane in the sense that all the PHEVs in that area have the same charging price. Inside an area, if the PHEVs are charging their batteries, the electricity load of this area and the daily payments of the owners will increase accordingly. All the electricity demands in the area constitute aggregated load to the entire grid, and different areas are independent of each other. In this multi-level power distribution system, aggregators are used for communication only and have a low cost, and this framework features a strong adaptability and scalability for widespread adoption of PHEVs. Figure 1 illustrates the PHEV charging scenario in the multi-level power distribution system, in which both power flow and signal flow are displayed.
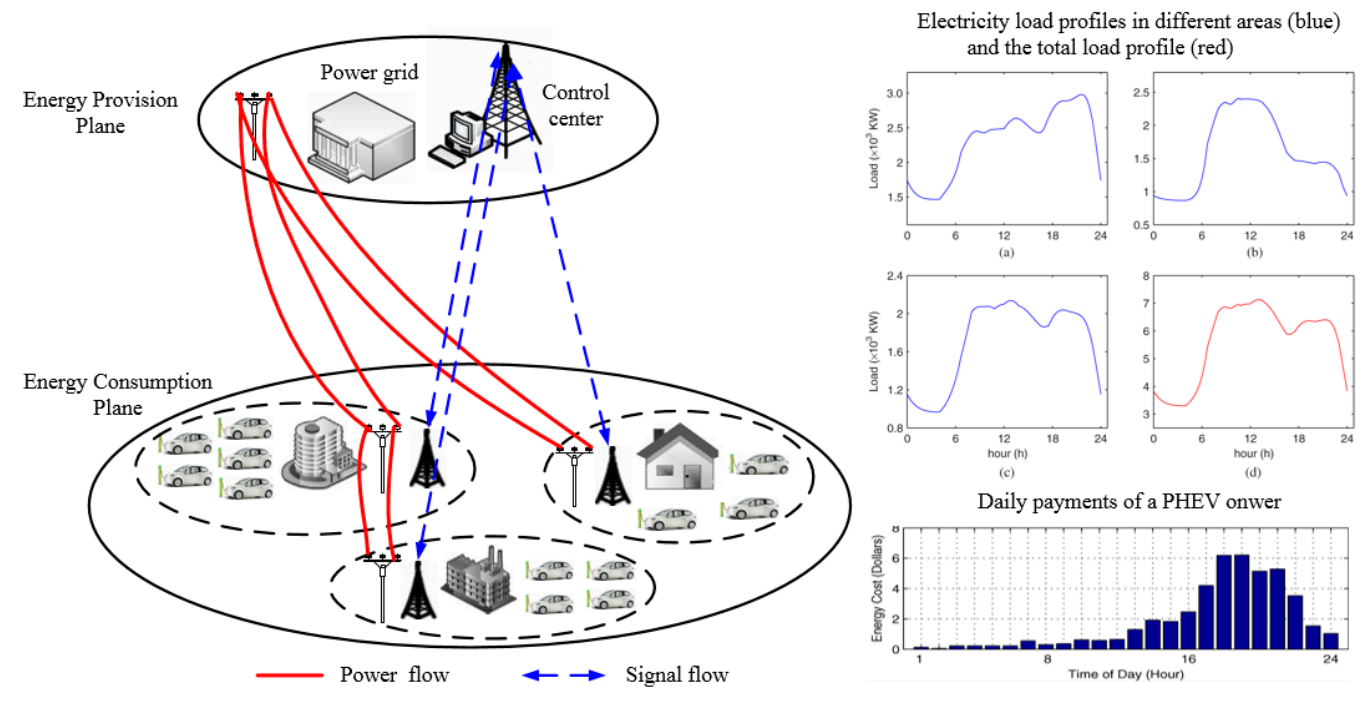

Figure 1. Overall framework of a power distribution system.

\subsection{Heterogeneous Information Network Modeling}

To facilitate PHEV charging in the multi-level power distribution system, a heterogeneous information network is modelled for this scenario. Since sensitive information about individuals, e.g., the vehicle owner's charging requirement and battery level, is prone to serious risks of privacy disclosure, it is essential to provide mechanisms for preserving data privacy while publishing useful 
information [15]. Thereby in the downlink, the control messages, such as price signals, are broadcast from the control centre to aggregators deployed in areas using cellular communications systems. Then the aggregator delivers the messages to the PHEVs using wireless local area network (LAN) communication systems with high security and favourable extensibility. In the uplink, the vehicular sensing data are upload in a reverse way to the aggregator accordingly, such as initial SOC, battery charge level, etc.

For the communication among a population of PHEVs in an area, suppose that each vehicle owner is equipped with an energy charging scheduler (ECS) [16], enabling it to communicate with its aggregator and neighbours. The digraph is employed to depict the communication topology among PHEVs. Let $k=1,2, \cdots, N$ denote the time index and $G(k)=(V, E(k))$ denote the topology of PHEVs at time $k$, where $V$ is the set of PHEVs and $E$ is the set of edges. For a PHEV $i \in V$, its in-neighbors and out-neighbors at time $k$ are denoted by $N_{i}^{+}(k):=\{j \in V:(j, i) \in E(k)\}$ and $N_{i}^{-}(k):=$ $\{j \in V:(i, j) \in E(k)\}$. The in-degree and out-degree of PHEV $i$ are denoted by $d_{i}^{+}(k):=\left|N_{i}^{+}(k)\right|$ and $d_{i}^{-}(k):=\left|N_{i}^{-}(k)\right|$, where $|\cdot|$ denotes the cardinality of a set. With the equipped ECS, we shall assume that the topology for the PHEVs in an area is strongly connected and that is there is a path between any two PHEVs. The heterogeneous information network for the multi-level power distribution system is presented in Figure 2.

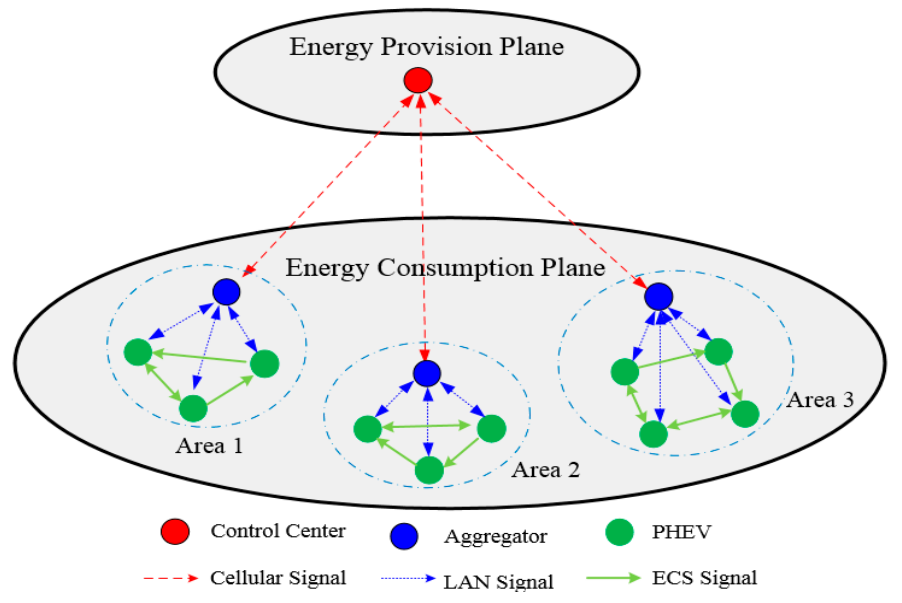

Figure 2. Heterogeneous information network for the multi-level power distribution system.

\subsection{PHEV Charging Modeling}

At present, it is common to use lithium-ion batteries in PHEVs due to their desirable load features and high-energy density. The state-of-charge (SOC) for the battery in a PHEV is defined as

$$
S(k)=\frac{C(k)}{C} \times 100 \%,
$$

where $C(\mathrm{kWh})$ is the capacity of battery energy and $C(k)(\mathrm{kWh})$ is the remaining capacity of battery energy at time $k$. The model for the $i$ th PHEV charging is given by

$$
S(k+1)=S(k)+\frac{x_{i}^{k} \cdot \Delta T}{C_{i}} \eta
$$


where $x_{i}^{k}$ is the charging power at time $k, \Delta T$ is the sampling interval, and $\eta$ is the coefficient assumed to be constant. The energy efficiency can reach a preferable level (i.e., over 95\%) using charging technology considering battery's lifespan [17]. Denoting

$$
a_{i}=\left\{\begin{array}{cc}
\frac{\Delta T}{C_{i}} \eta & \text { if } C_{i}>0 \\
0 & \text { if } C_{i}=0
\end{array}\right.
$$

Equation (2) is rewritten as

$$
S(k+1)=S(k)+a_{i} x_{i}^{k}
$$

which is applicable for the PHEVs whether they are charging or not.

\section{Problem Formulation}

In this paper, we focus on a multi-objective coordinated charging scheme of PHEVs that allows certain desired capabilities of the aggregate load both on the power grid and the vehicle owners. In detail, the multiple objectives are performed with different priorities. From the view of the energy provider, it is aimed at stabilizing the power load with a higher priority via controlling the aggregated power for PHEV charging based on the electricity demands. On the basis of that, the total minimum cost of a group of PHEVs in an area is achieved with a lower priority by optimally allotting the aggregated power, while satisfying their charging requirements by the specified time.

We shall assume that the vehicle owners are willing to participate in the coordination of their energy usage provided that they are provided with sufficient incentives such as less payments. Suppose that there are $n$ PHEVs in a set $V$ of an area, and the charging horizon is evenly divided into $N$ time slots, denoted by $k=1,2, \cdots, N$. For PHEV $i \in V$, the charging vector is defined as

$$
x_{i}=\left[x_{i}^{1}, \cdots, x_{i}^{k}, \cdots, x_{i}^{N}\right]^{\mathrm{T}},
$$

where $x_{i}^{k}$ is the $i$-th PHEV charging power at time $k$. The charging cost of buying an $x_{i}^{k}$ unit of energy from the power grid is denoted by $f(x)$ as

$$
f(x)=a_{i}^{k}\left(x_{i}^{k}\right)^{2}+b_{i}^{k} x_{i}^{k}+c_{i}^{k}
$$

with $a_{i}^{k}>0$ and $b_{i}^{k}, c_{i}^{k} \in \mathbb{R}$. For simplicity of expression, an equivalent function is used in this paper as

$$
f(x)=\frac{\left(x_{i}^{k}-\alpha_{i}^{k}\right)^{2}}{2 \beta_{i}^{k}}+\gamma_{i}^{k},
$$

with constants $\alpha_{i}^{k} \leq 0, \beta_{i}^{k}>0$ and $\gamma_{i}^{k} \geq 0$. The corresponding incremental function of $f(x)$ is

$$
f^{\prime}(x)=\frac{d f(x)}{d x}=\frac{x_{i}^{k}-\alpha_{i}^{k}}{\beta_{i}^{k}}
$$

For the objectives with different priorities, if $F(x)$ has a higher priority than $G(x)$, then we express it as

$$
F(x)>G(x)
$$

The coordinated charging problem of PHEVs in the multi-level power distribution system is formulated as follows. 
- $\quad$ Objectives

$$
\left\{\begin{array}{c}
\min F(x)=\sum_{k=1}^{N}\left(\sum_{i=1}^{n}\left(x_{i}^{k}+q_{i}^{k}\right)-\xi\right)^{2}, \\
\min G(x)=\sum_{i=1}^{n} \sum_{k=1}^{N} \frac{\left(x_{i}^{k}-\alpha_{i}^{k}\right)^{2}}{2 \beta_{i}^{k}} .
\end{array}\right.
$$

- Constraints

- $\quad$ Inequality constraints on $x_{i}^{k}$ as

$$
0 \leq x_{i}^{k} \leq \overline{x_{i}}, \forall i, k
$$

- $\quad$ Equality constraints on $x_{i}^{k}$ as

$$
\sum_{k=1}^{N} x_{i}^{k}=b_{i}, \quad \forall i .
$$

- Inequality priority constraint on objectives as

$$
F(x)>G(x)
$$

where $\xi$ is the ideal flat power curve; $q_{i}^{k}$ is the base electricity demand of an area that is known to the aggregator; $\overline{x_{i}}$ is the maximum charge power of the $i$-th PHEV and $b_{i}$ is the charging requirement of the $i$-th PHEV set by the owner, which should be satisfied at the end of charging.

The vehicle owner intends to charge their vehicle from initial SOC $S_{i}(0)$ to target SOC $S_{i}^{*}$. From Equation (3), it can be inferred that

$$
S_{i}^{*}=S_{i}(0)+a_{i} \sum_{k=1}^{N} x_{i}^{k} .
$$

Thereby $b_{i}$ is given by

$$
b_{i}=\left(S_{i}^{*}-S_{i}(0)\right) / a_{i}
$$

Since the cost function $F(x)$ denotes the fluctuation between the total demand curve and the ideal flat power curve. It is verified that $F(x)=0$ if and only if the aggregate power curve is flat over $k$, and $\xi$ is given by

$$
\xi=\frac{1}{N}\left(\sum_{i=1}^{n} b_{i}+\sum_{k=1}^{N} \sum_{i=1}^{n} q_{i}^{k}\right) .
$$

Remark 1. In the multi-objective optimization problem (8)-(11), we are motivated by the constraints on objectives with priority to deal with single-objective optimization problems with different priorities under the system's constraints. When only $F(x)$ or $G(x)$ is considered, the constraint shown in Equation (11) becomes redundant, making Equations (8)-(11) easier to solve.

\section{Main Results}

In this section, we give a transformation for the problem (8)-(11) based on the priorities of objective functions. Then we propose a hierarchical optimal algorithm to solve the transformed problem, and present the convergence analysis for our algorithm. Finally, a practical case is considered, in which PHEVs arrive and leave randomly and the hierarchical algorithm with a moving horizon method is developed. 


\subsection{Primal Problem Transformation}

Based on the priorities of the objective functions, we transform the problem (8)-(11) into two single-objective optimization problems. First, we aim to shift the load demand by controlling the aggregated power for PHEVs charging in the area. Then we focus on achieving the total minimum cost for the vehicle owners in the area by optimally allotting the aggregated power for each vehicle, while satisfying their charging requirements by the specified time.

From the equality constraints (Equation (10)), we have

$$
\sum_{i=1}^{n} \sum_{k=1}^{N} x_{i}^{k}=\sum_{i=1}^{n} b_{i}
$$

which is equivalent to

$$
\sum_{k=1}^{N} \sum_{i=1}^{n} x_{i}^{k}=\sum_{i=1}^{n} b_{i}
$$

Let $d^{k}$ denote the aggregated power provided for PHEVs charging in the area at time $k$ as

$$
d^{k}=\sum_{i=1}^{n} x_{i}^{k}, \quad \forall k .
$$

Thus the problem (8)-(11) can be transformed into the optimal load-shifting problem as

$$
\begin{gathered}
\min \sum_{k=1}^{N}\left(d^{k}+\sum_{i=1}^{n} q_{i}^{k}-\xi\right)^{2} \\
\text { s.t. }\left\{\begin{array}{c}
0 \leq d^{k} \leq \sum_{i=1}^{n} \overline{x_{i}}, \forall k, \\
\sum_{k=1}^{N} d^{k}=\sum_{i=1}^{n} b_{i},
\end{array}\right.
\end{gathered}
$$

and the operation cost minimization problem as

$$
\begin{gathered}
\min \sum_{i=1}^{n} \sum_{k=1}^{N} \frac{\left(x_{i}^{k}-\alpha_{i}^{k}\right)^{2}}{2 \beta_{i}^{k}} \\
\text { s.t. }\left\{\begin{array}{c}
0 \leq x_{i}^{k} \leq \overline{x_{i}}, \forall i, k, \\
\sum_{k=1}^{N} x_{i}^{k}=b_{i}, \forall i, \\
\sum_{i=1}^{n} x_{i}^{k}=d^{k}, \forall k,
\end{array}\right.
\end{gathered}
$$

where $d^{k}$ and $x_{i}^{k}$ are the optimization variables of Equations (18) and (19), respectively.

Remark 2. The transformed single-objective optimization problems (18) and (19) differ from the primal problem (8)-(11) both in the optimization variables and the constraints. The inequality priority constraints on objectives are absent due to the introduction of the variable $d^{k}$, making the problems (18) and (19) easier to solve than the primal problem. It is reasonable to expect that the objective in problem (18) with higher priority also has a higher level of satisfaction than that in problem (19).

\subsection{Hierarchical Algorithm}

Now we present our hierarchical optimal algorithm, combining the water-filling-based algorithm with the consensus-based method to solve problems (18) and (19). 


\subsubsection{Water-Filling-Based Algorithm}

First, we apply the water-filling-based algorithm to solve the optimal load shifting problem (18). Let $q_{k}=\sum_{i=1}^{n} q_{i}^{k}, d_{\max }^{k}=\sum_{i=1}^{n} \overline{x_{i}}$ and $b=\sum_{i=1}^{n} b_{i}$. Following the Lagrangian multiplier method, for the optimal load-shifting problem (18), the Lagrange function can be constructed as

$$
L(d, \lambda)=\sum_{k=1}^{N}\left(d^{k}+q^{k}-\xi\right)^{2}+2 \lambda\left(\sum_{k=1}^{N} d^{k}-b\right) .
$$

where the first term is the objective function of Equation (18); the second term is designed to satisfy the equality constraint in Equation (18) by introducing the Lagarange multiplier $\lambda$, and the constant of 2 in Equation (20) is designed for convenience. By differentiating Equation (20) with respect to $d^{k}$ and setting the result to be zero, we have

$$
d^{k}+q^{k}-\xi+\lambda=0
$$

The equipower level [18], is denoted by $\delta=\xi-\lambda$ and is independent of $k$. Equation (21) is rewritten as

$$
d^{k}+q^{k}=\delta .
$$

It should be pointed out that Equation (22) is obtained from the constructed Lagrange function (20) by differentiating Equation (20) with respect to $d^{k}$. The equipower level $\delta$ is a constant which is set as $\min _{k} q^{k}$ in the initialization. Moreover, the optimality condition (22) does not take into account the inequality constraint $0 \leq d^{k} \leq d_{\max }^{k}$. If this constraint is taken into account, Equation (22) becomes the following: there exists an equipower level constant $\delta$ such that either Equation (22) holds or

$$
d^{k}=0 \text { and } d^{k}+q^{k} \geq \delta
$$

or

$$
d^{k}=d_{\max }^{k} \text { and } d^{k}+q^{k} \leq \delta .
$$

The water-filling-based algorithm is presented in Algorithm 1. In Algorithm 1, D(.) is the projection operation designed to confine $d^{k}$ in the interval $\left[0, d_{\max }^{k}\right]$, that is

$$
D\left(d^{k}\right)=\left\{\begin{array}{cc}
d_{\max }^{k} \quad \text { if } d^{k}>d_{\max }^{k} \\
d^{k} & \text { if } 0 \leq d^{k} \leq d_{\max }^{k} \\
0 & \text { if } d^{k}<0 .
\end{array}\right.
$$

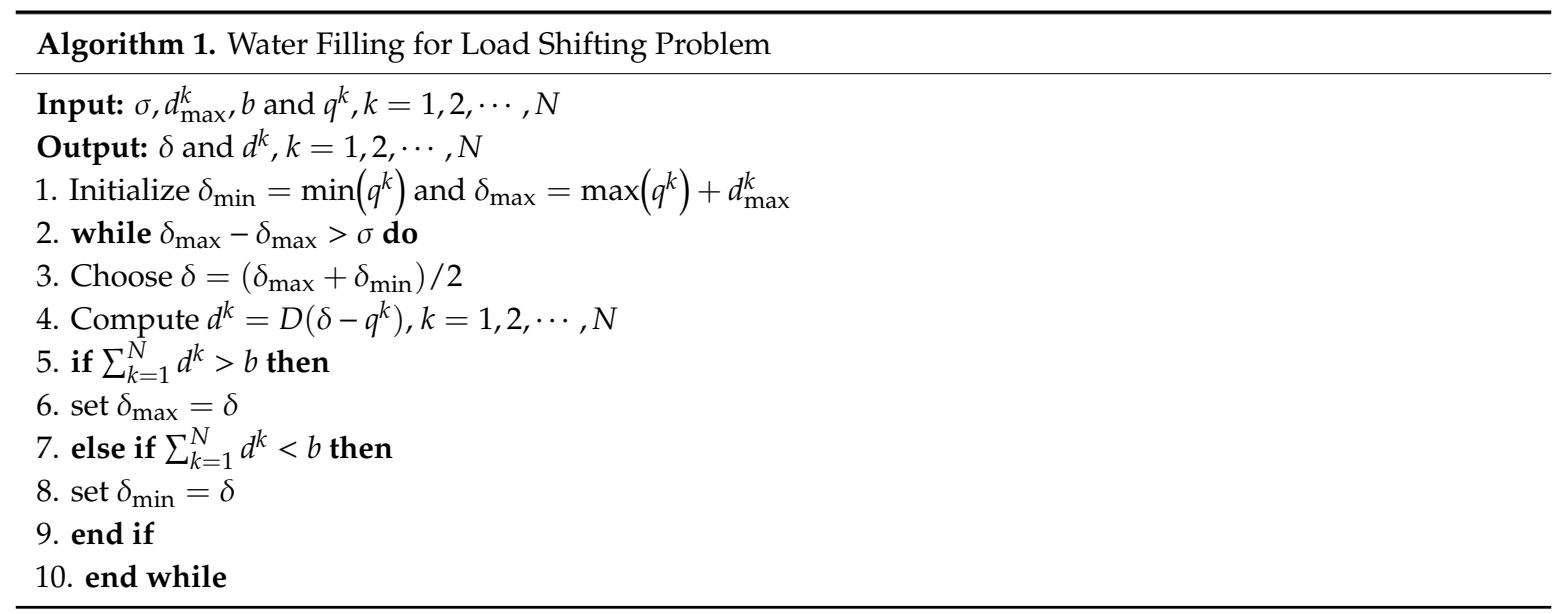


Remark 3. Algorithm 1 is interpreted based on the water-filling-based algorithm which is applied to the case of a single PHEV in a smart grid scenario [18]. However, our work is quite different from [18] in terms of both optimization variables and constraints. In addition, the control center computes and communicates with the aggregator at each time slot, so is not required to be computationally powerful, as the computational burden will be evenly shared by each time slot in the multi-level power distribution system.

\subsubsection{Consensus-Based Method}

The consensus-based method is proposed in our previous work [19], which only requires local information and all nodes merely communicate with their neighbors to solve a dynamic resource allocation problem. The definition of the updated variables (i.e., $\lambda_{i}^{k}, s_{i}^{k}$, and $\varphi_{i}^{k}$ ) for iteration can be referred to [19]. We apply this method to solve problem (19) which is interpreted as follows.

Step 1: Initializing the variables to be updated through the Equation (26).

Step 2: Let each PHEV $i$ at time slot $k$ in the area have its own copy of the Lagrange multiplier $\lambda_{i}^{k}$ to satisfy the equality aggregated power constraint in problem (19). Then we update $\lambda^{k}=\left[\lambda_{1}^{k}, \cdots, \lambda_{n}^{k}\right]^{\mathrm{T}}$ such that $\lambda_{i}^{k}(i=1,2, \cdots, n)$ reaches consensus according to the consensus-based iteration (27).

Step 3: Let each PHEV $i$ have a dual multiplier $v_{i}$ which is used to satisfy the equality charging requirement constraint in problem (19), and update the dual multiplier according to the consensus-based iterations (28).

Step 4: Each PHEV $i$ at time slot $k$ update its estimated value according to the nonlinear projection (Equation (28)), mapping $x_{i}^{k}$ to the interval $\left[0, \overline{x_{i}}\right]$ to satisfy the inequality state constraints in problem (19).

Step 5: Due to the nonlinear projection map, the estimated vector $x_{i}^{k}=\left[x_{1}^{k}, \cdots, x_{n}^{k}\right]^{\mathrm{T}}$ at each step may not be a feasible solution to problem (19). To overcome this issue, let each PHEV $i$ at time slot $k$ be associated with a surplus variable $s_{i}^{k}$ to temporarily store the resulting deviation. Then update $s_{i}^{k}=\left[s_{1}^{k}, \cdots, s_{n}^{k}\right]^{\mathrm{T}}$ which will be averaged among its neighbors such that $s^{k}$ will vanish gradually.

These ideas are summarized in Algorithm 2.

Remark 4. Algorithm 2 is distributed due to the fact that the algorithm only requires local information from its neighbors in an area. That is the $i$-th PHEV at time slot $k$ receives $\lambda_{j}^{k}(t)$ and $s_{j}^{k}(k) / d_{j}^{-}(k)$ from its in-neighbors, and sends $\lambda_{i}^{k}(t)$ and $s_{i}^{k}(k) / d_{i}^{-}(k)$ to its out-neighbors. This means all the items in Algorithm 2 can be obtained in a distributed way.

Remark 5. The proposed hierarchical algorithm combines the water-filling-based algorithm and the consensus-based optimization method together. The transformed single-objective optimization problems (18) and (19) can be solved successively and efficiently by the hierarchical algorithm, in which the optimal solution to problem (18) is used for solving problem (19), and its implementation is convenient in the multi-level power distribution system. 


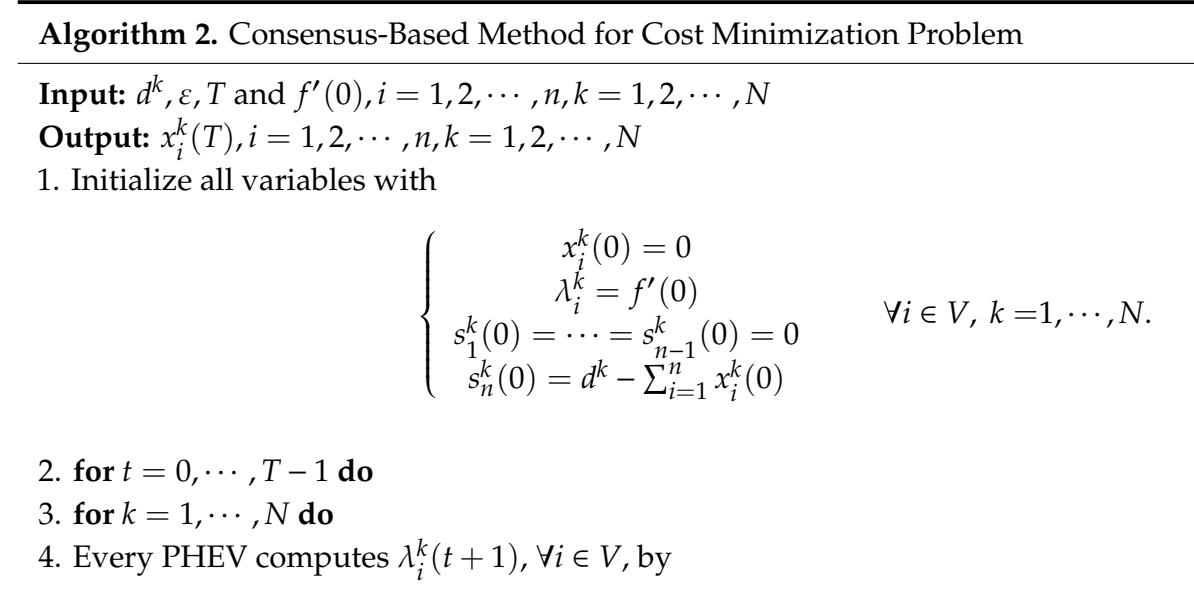

$$
\lambda_{i}^{k}(t+1)=\lambda_{i}^{k}(t)+\sum_{j \in N_{i}^{+}(k)} \frac{\lambda_{j}^{k}(t)-\lambda_{i}^{k}(t)}{d_{i}^{+}(k)}+\varepsilon s_{i}^{k}(t)
$$

5. end for

6. for $i=1, \cdots, n$ do

7. PHEV $i$ computes $v_{i}(t+1)$ by solving

$$
\sum_{k=1}^{N} \varphi_{i}^{k}\left(\lambda_{i}^{k}+v_{i}\right)-b_{i}=0
$$

8. PHEV $i$ computes $x_{i}^{k}(t+1), k=1,2, \cdots, N$, by

$$
x_{i}^{k}(t+1)=\varphi_{i}^{k}\left(\lambda_{i}^{k}(t+1)+v_{i}(t+1)\right)
$$

9. end for

10. for $k=1, \cdots, N$ do

11. Every PHEV computes $s_{i}^{k}(t+1), \forall i \in V$, by

$$
s_{i}^{k}(t+1)=\frac{s_{i}^{k}(t)}{d_{i}^{-}(k)}+\sum_{j \in N_{i}^{+}(k)} \frac{s_{j}^{k}(t)}{d_{j}^{-}(k)}-\left(x_{i}^{k}(t+1)-x_{i}^{k}(t)\right)
$$

\section{2. end for}

13. if $\left|\sum_{i=1}^{n} \sum_{k=1}^{N} s_{i}^{k}(t+1)\right|>0$ then

14. set $t+1=t$

15. else if $\left|\sum_{i=1}^{n} \sum_{k=1}^{N} s_{i}^{k}(t+1)\right|=0$ then

16. break

17. end if

18. end for

\subsection{Convergence Analysis}

In this subsection, the convergence analysis of the proposed hierarchical algorithm is given.

Theorem 1. The solution to the multi-objective optimization problem (8)-(11) given by the hierarchical algorithm is optimal when $\sigma=0$ and $\varepsilon$ is sufficiently small.

The proof of Theorem 1 is divided into two steps. First, we prove that the solution to problem (18) is optimal when $\sigma=0$ using the water-filling-based algorithm. After that, we prove that the solution 
to problem (19) is optimal using the consensus-based method if $\varepsilon$ is sufficiently small. Lemma 1 is about the solution to problem (18) is optimal using water-filling-based algorithm for a single PHEV.

Lemma 1. The optimal solution to problem

$$
\begin{gathered}
\min \sum_{k=0}^{N-1}(p(k)+q(k)-\xi)^{2} \\
\text { s.t. }\left\{\begin{array}{l}
0 \leq p(k) \leq p_{\max } \\
\sum_{k=0}^{N-1} p(k)=b_{0}
\end{array}\right.
\end{gathered}
$$

is given by

$$
p^{*}(k)=P\left[-\lambda^{*}-q(k)\right], \quad \forall k,
$$

where $p(k)$ is the optimization variable representing the charging power of a single PHEV; $q(k)$ is the base demand; $\xi$ is the ideal flat power curve; $b_{0}$ is the charging requirement of the PHEV over N-period charging horizon; $p^{*}(k)$ is the optimal solution to the problem (31); $\lambda^{*}$ is the optimal Lagrange multiplier, and $P[\cdot]$ is

$$
P[x(k)]=\left\{\begin{array}{cc}
p_{\max } & \text { if } x(k)>p_{\max } \\
x(k) & \text { if } 0 \leq x(k) \leq p_{\max } \\
0 & \text { if } x(k)<0 .
\end{array}\right.
$$

Proof of Lemma 1. See the proof of Lemma 1 in [20].

Comparing the problems (18) and (31), both on optimization variable and constraints, it is verified that the solution to problem (18) is optimal using the water-filling-based algorithm when $\sigma=0$. Lemma 2 is about the optimal consensus-based iteration for dynamic resource allocation over strongly connected topology [19].

Lemma 2. The consensus-based algorithm is stable and all the variables converge to the optimal solution if the positive constant $\varepsilon$ is sufficiently small.

Proof of Lemma 2. See the proof of Theorem 1 in [19].

Now we give the proof of Theorem 1.

Proof of Theorem 1. The multi-objective optimization problem (8)-(11) is transformed into two sub-optimizations, problems (18) and (19), following the objective functions' priorities. From Lemma 1 , we conclude that the solution to problem (18) given by Algorithm 1 is optimal when $\sigma=0$. After Algorithm 1 converges, the solution to problem (19) given by Algorithm 2 is optimal when $\varepsilon$ is sufficiently small following Lemma 2 . This establishes Theorem 1.

Remark 6. Due to the fact that the solution to problem (18) is used for solving problem (19), we point out that the solution to problem (19) given by Algorithm 2 is optimal based on the optimal solution to problem (18) given by Algorithm 1.

\subsection{Hierarchical Algorithm with Moving Horizon}

The proposed hierarchical algorithm assumes that all PHEVs have the same entry and exit times in an area, which may be unrealistic in practice. In this subsection, we consider a practical case where PHEVs can arrive and leave randomly. A moving horizon method is adopted to deal with this case. A moving horizon method means that only the first control sample will be implemented 
after computation of the optimal control sequence. Then the horizon is shifted one sample and the optimization is restarted with new information of the measurements subsequently.

To this end, a hierarchical algorithm with moving horizon is proposed. The optimal load-shifting problem (18) and the operation cost minimization problem (19) need some adjustments on objective function in this case as

$$
\begin{gathered}
\min \sum_{k=t}^{K-1}\left(d^{k}+\sum_{i=1}^{n} q_{i}^{k}-\xi(k)\right)^{2} \\
\text { s.t. }\left\{\begin{array}{c}
0 \leq d^{k} \leq \sum_{i=1}^{n} \overline{x_{i}}, \forall k \\
\sum_{k=1}^{N} d^{k}=\sum_{i=1}^{n} b_{i},
\end{array}\right.
\end{gathered}
$$

and

$$
\begin{gathered}
\min \sum_{i=1}^{n} \sum_{k=t}^{K-1} F_{i}^{k}\left(x_{i}^{k}\right) \\
\text { s.t. }\left\{\begin{array}{l}
0 \leq x_{i}^{k} \leq \overline{x_{i}}, \forall i, k, \\
\sum_{k=1}^{N} x_{i}^{k}=b_{i}, \forall i, \\
\sum_{i=1}^{n} x_{i}^{k}=d^{k}, \forall k,
\end{array}\right.
\end{gathered}
$$

where $t$ is the present time of the optimization start; $K=\max \left(K_{1}, \cdots, K_{n}\right)$ and $K_{i}$ is the charging horizon of the $i$-th PHEV at time slot $k$ in the area. $K_{i}$ is set to zero if PHEV $i$ does not arrive. Not until a new PHEV arrives with a late entry time can $K$ change. Denoted by $\xi(k)$ the ideal flat power curve at time $k$ in the group is given by

$$
\xi(k)=\frac{\sum_{i=1}^{n}\left(b_{i}-b_{i}(t-1)\right)+\sum_{k=t}^{K-1} \sum_{i=1}^{n} q_{i}^{k}}{K-t} .
$$

The hierarchical algorithm with moving horizon method is presented in Algorithm 3.

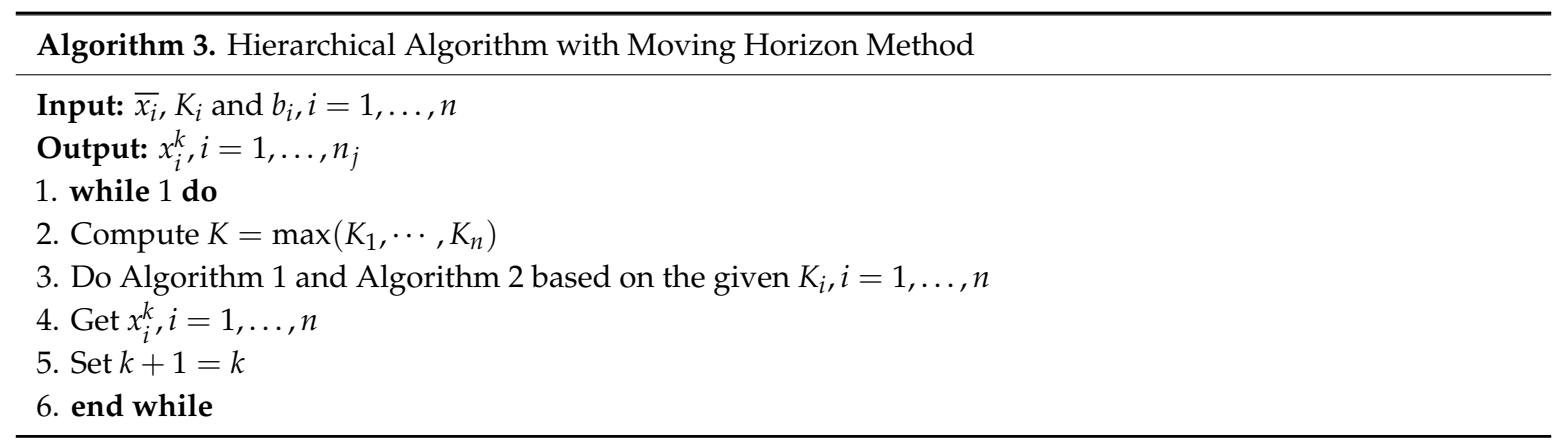

Remark 7. Algorithm 3 is designed for solving Equations (34) and (35) online, in which the result is optimal in the sense for a given $K_{i}$. In Algorithm 3, $x_{i}^{k}$ is updated at each time slot $k$ in the group theoretically. To reduce computational burden, $x_{i}^{k}$ is updated when another PHEV arrives with a later time in practice.

\section{Simulation}

In this section, the effectiveness of the proposed algorithm is verified through numerical simulations. First, a numerical simulation is given to illustrate Algorithms 1 and 2. Then, a general case is considered to illustrate Algorithm 3. Finally, power consumption data of an area is used to show the desirable performance of our algorithm.

\subsection{Simulation for Algorithms 1 and 2}

In this simulation, we consider there are four PHEVs in an area of the power distribution system. The simulation parameters are given in Table 1. The unit for power is $\mathrm{kW}$ and unit for energy is $\mathrm{kWh}$. In this simulation, the start and exit times for all PHEVs are assumed to be 1 and 168, which 
corresponds to 18:00 and 18:00 (on another day). The sampling period is 7 samples/h and there are 168 samples for $24 \mathrm{~h}$. The positive parameter $\varepsilon$ is set to 0.55 .

Table 1. Parameters of four plug-in hybrid electric vehicles (PHEVs).

\begin{tabular}{ccccc}
\hline PHEV No. & Max Power & Energy Need & Exit Time & Base Demand \\
\hline 1 & 3.5 & 28 & 168 & $0.25 \mathrm{q}$ \\
2 & 6 & 32 & 168 & $0.15 \mathrm{q}$ \\
3 & 3.9 & 25 & 168 & $0.4 \mathrm{q}$ \\
4 & 4.6 & 30 & 168 & $0.2 \mathrm{q}$ \\
\hline
\end{tabular}

In Figure 3, the base demand (non-PHEVs) is given by the blue curve, which is from [21]. The computed aggregated power references $d^{k}, k=1, \ldots, 168$, are given by the red curve using Algorithm 1 , and the green curve represents the total aggregate power curve which has been flattened maximally, achieving valley filling. Figure 4 plots a balanced power allocation for the PHEVs from Algorithm 2. Figure 5 plots the error norm $\left\|x(t)-x^{*}\right\|$, converging to zero with respect to the iterative steps, demonstrating that Algorithm 2 is able to achieve the optimal solution for this scenario. Without coordinated charging, each PHEV charges its battery with maximum power as soon as the PHEV arrives. Compared with uncoordinated charging, Figure 6 shows the lower daily charging cost of each PHEV has been achieved using coordinated charging scheduling.

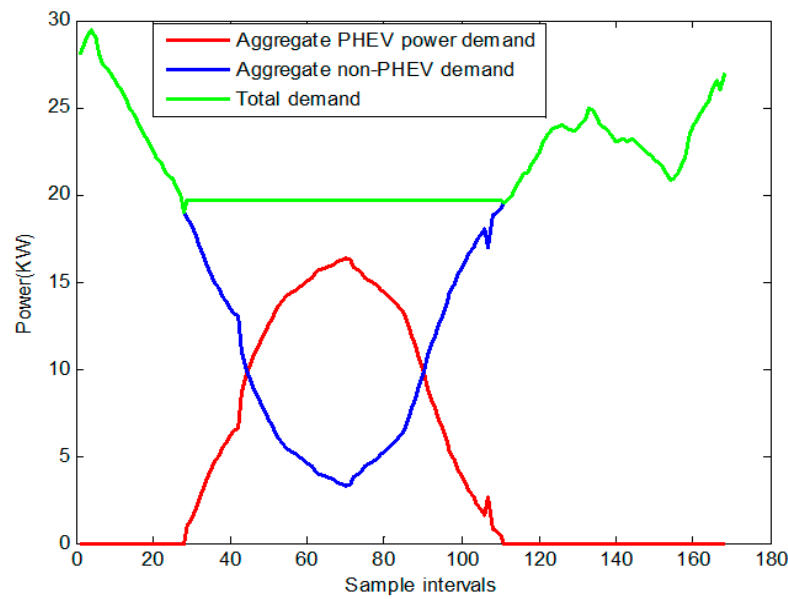

Figure 3. Power curve from Algorithm 1.

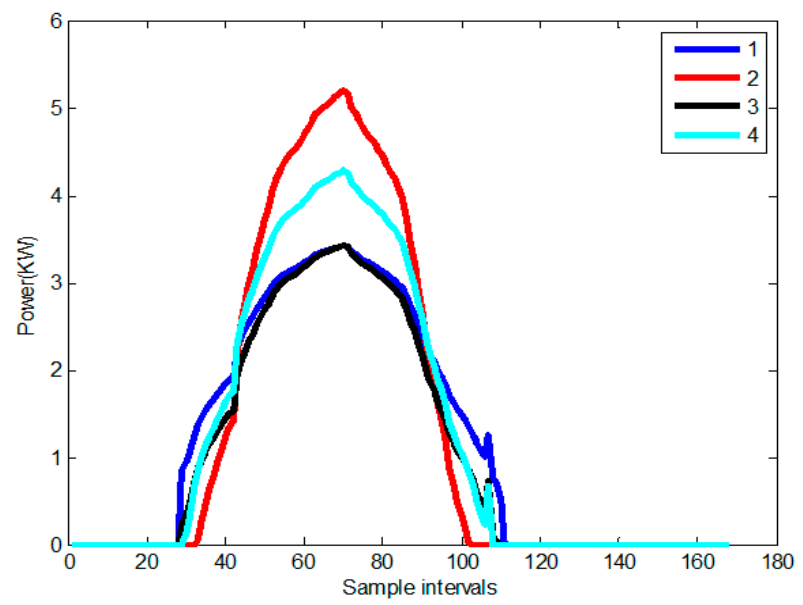

Figure 4. Power allocation for the PHEVs from Algorithm 2. 


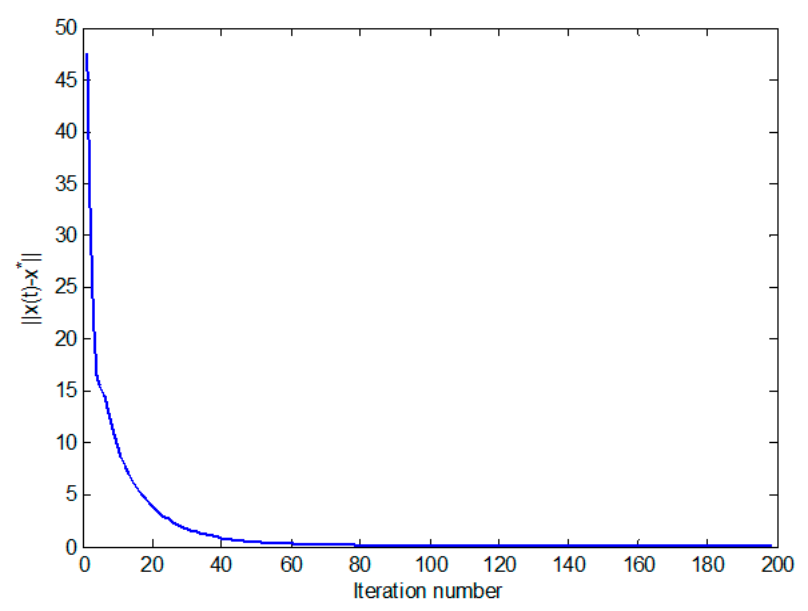

Figure 5. Convergence of Algorithm 2.

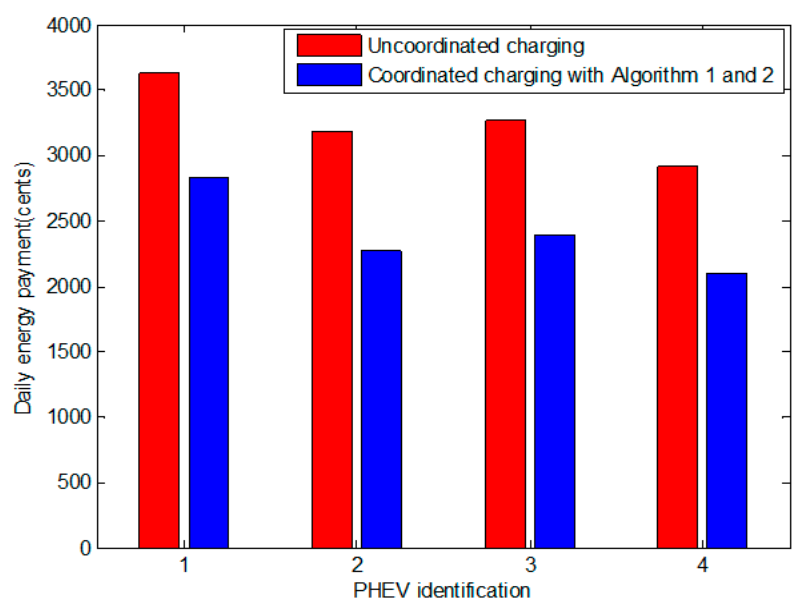

Figure 6. Daily energy cost for the PHEVs.

\subsection{Simulation for Algorithms 3}

In this subsection, a general case is considered for the random arrival and departure of PHEVs to illustrate Algorithm 3. The simulation parameters for this case are given in Table 2. The positive parameter $\varepsilon$ is set to 0.45 .

Table 2. Parameters of four PHEVs in the random arrival and departure case.

\begin{tabular}{ccccc}
\hline PHEV No. & Max Power & Energy Need & Entry Time & Exit Time \\
\hline 1 & 4.5 & 25 & 1 & 84 \\
2 & 6 & 35 & 1 & 108 \\
3 & 4.5 & 30 & 32 & 98 \\
4 & 5 & 32 & 35 & 126 \\
\hline
\end{tabular}

Figure 7 illustrates that the total aggregate power curve can be flattened maximally, achieving valley filling even if PHEV 3 and PHEV 4 arrive later and leave with different times. Figure 8 plots the power allocation for each PHEV using Algorithm 3 for this case. In Figure 9, we can see that the error norm $\left\|x(t)-x^{*}\right\|$ converges to zero over three online optimizations with respect to the iterative steps, showing that Algorithm 3 achieves the optimal solution for this case. Compared with uncoordinated charging in this case, coordinated charging scheduling clearly reduces the daily charging cost of each PHEV (see Figure 10). 


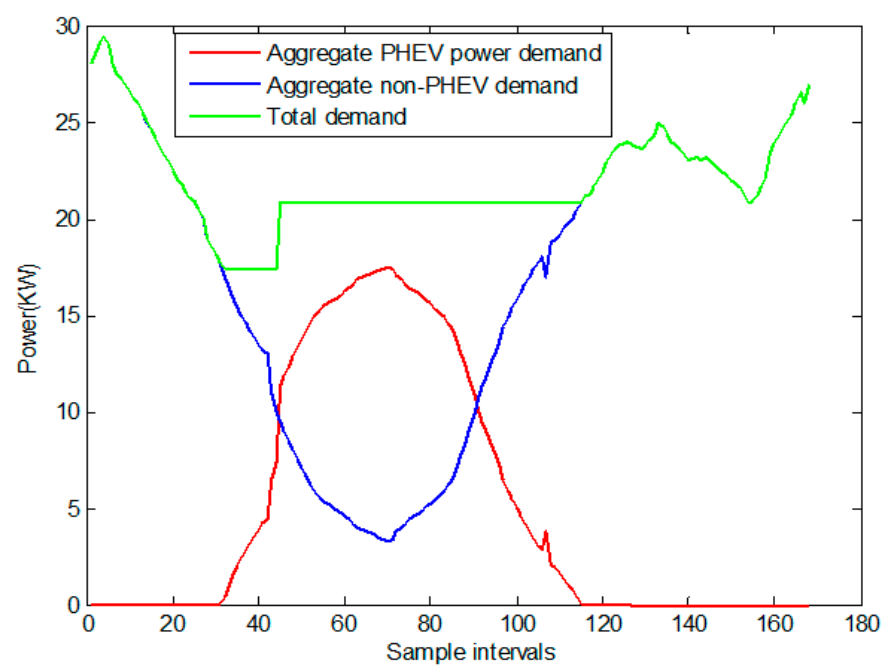

Figure 7. Power curve from Algorithm 3.

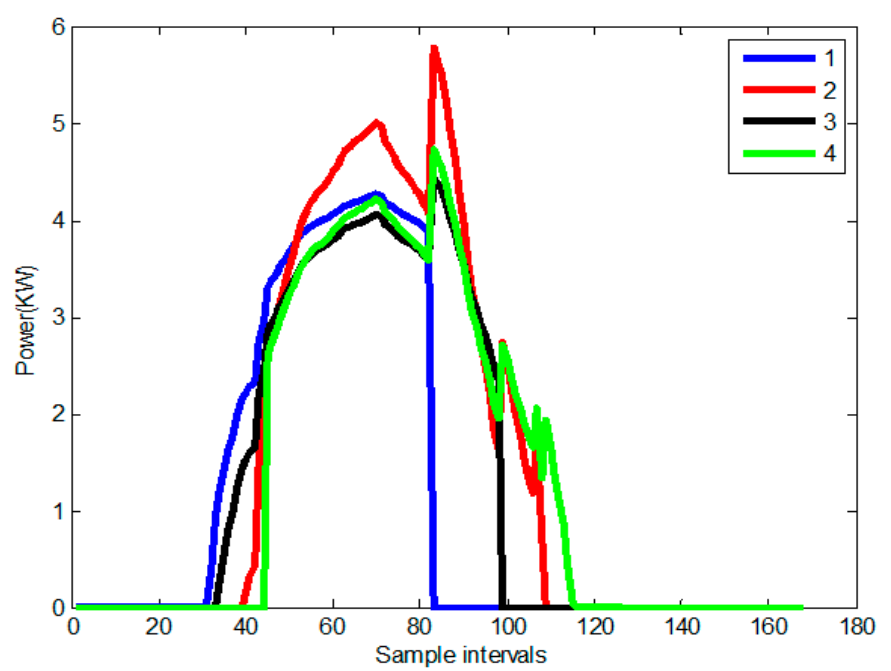

Figure 8. Power allocation for the PHEVs from Algorithm 3.

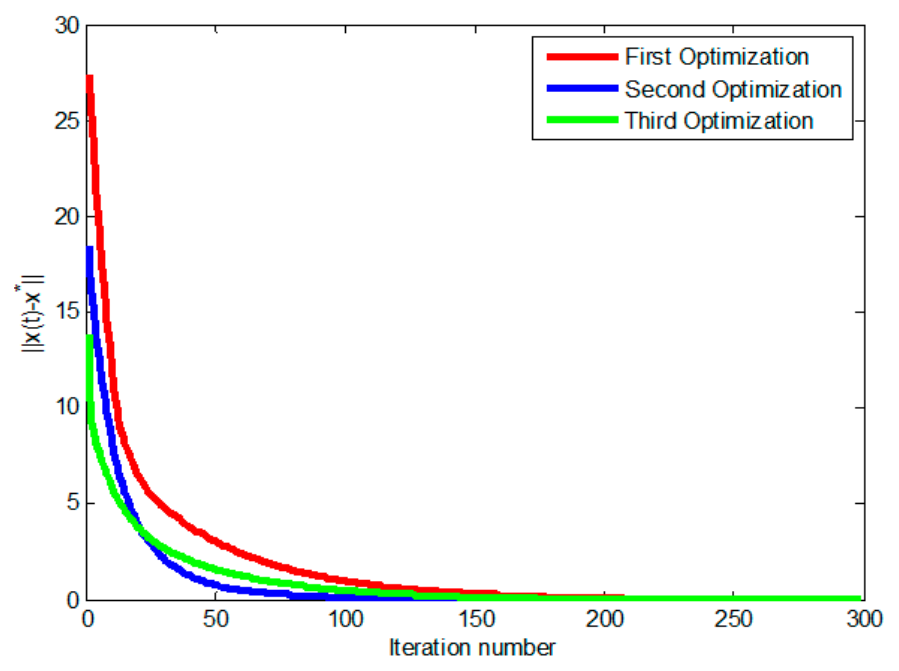

Figure 9. Convergence of Algorithm 3. 


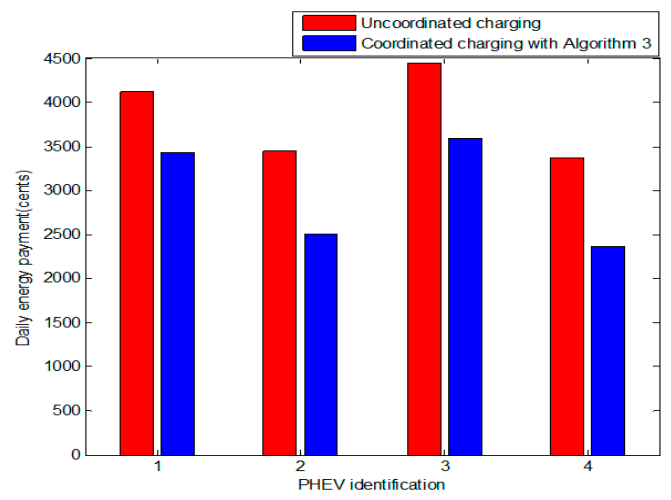

Figure 10. Daily energy cost for four PHEVs in the random arrival and departure case.

\subsection{Simulation Using Power Consumption Data}

In this subsection, power consumption data is used to show the desirable performance of our algorithm. The simulation parameters of four PHEV models are given in Table 3, the data in which was sources from [22,23]. The positive parameter $\varepsilon$ is set to 0.35 in this example.

Table 3. Parameters of four PHEV models.

\begin{tabular}{cccccc}
\hline PHEV Models & Battery Size & Energy Need & Max Power & Entry Time & Exit Time \\
\hline GM Chevy Volt & 16 & 8 & 3.84 & $18: 00$ & $06: 00$ \\
Tesla MODEL S & 60 & 40 & 10 & $18: 00$ & $09: 00$ \\
Nissan Leaf & 24 & 15 & 6.6 & $23: 00$ & $08: 00$ \\
BMW Mini E & 35 & 17 & 11.52 & $24: 00$ & $18: 00$ \\
\hline
\end{tabular}

Figure 11 shows the simulation results using power consumption data for a household with a Nissan Leaf. As depicted in Figure 11a, the base demand curve has a similar pattern to [21]. From Figure 11a, we can see that it is a $24 \mathrm{~h}$ base demand data and there are 1440 samples with the sample period of $1 \mathrm{~min}$. Figure $11 \mathrm{~b}$ is emulated with a low-pass filter based on the data from Figure 11a. Figure 11c is the power allocation curve of the PHEV using Algorithm 3. Figure 11d is the total aggregate power curve and we can see that there is no new peak load. Figure 12 shows that there are several new peak loads (e.g., around the 100th sample and 400th sample) without Algorithm 3. Compared with that, there is no new peak load using Algorithm 3. Figure 13 shows that the desirable low-cost performance is achieved using our algorithm, contributing to reduced energy consumption cost for each vehicle owner and providing the peak-shifting service for the power grid.
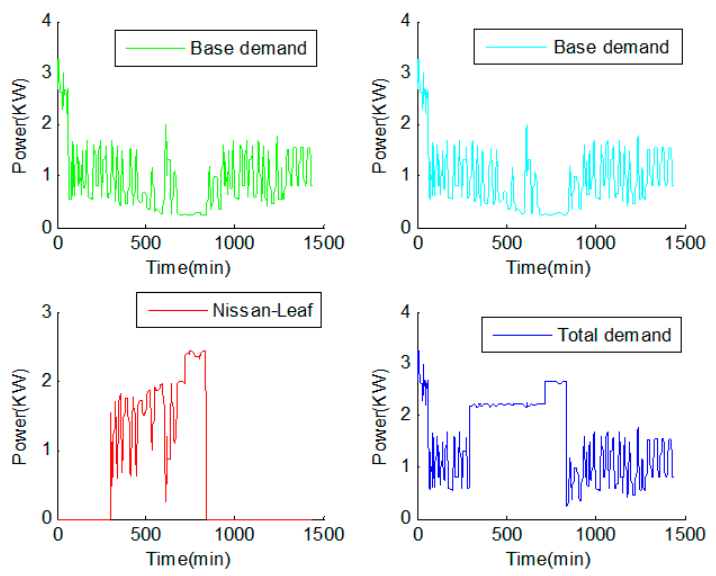

Figure 11. Simulation results using power consumption data for a household with a Nissan Leaf. (a) Realistic base demand curve. (b) Filtered base demand curve. (c) Power allocation for the PHEV from Algorithm 3. (d) Total aggregate power curve. 

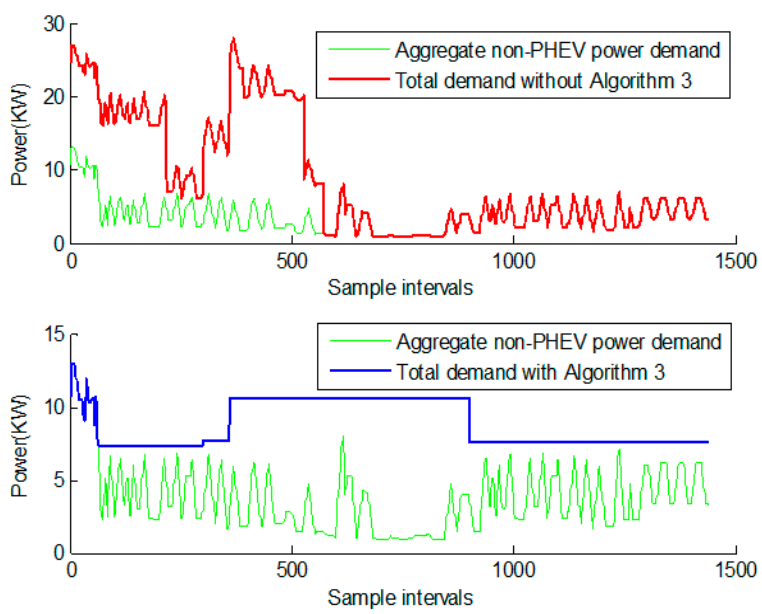

Figure 12. Power curves of the distribution system with and without Algorithm 3.

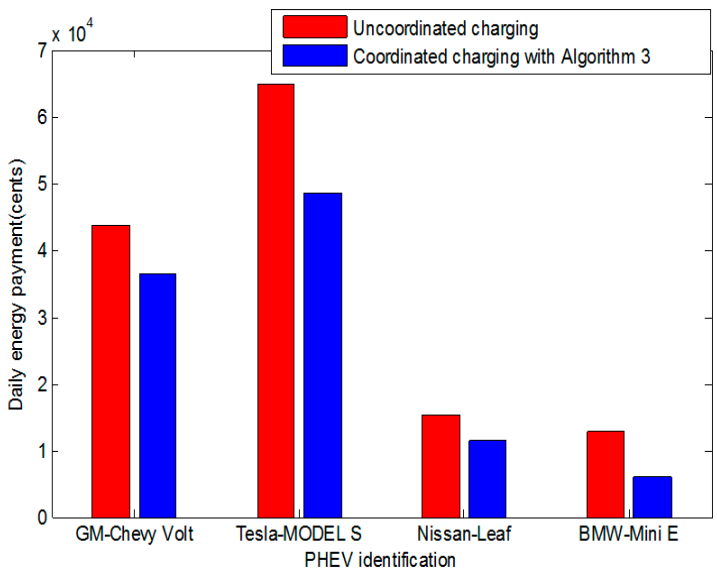

Figure 13. Daily energy costs for the PHEVs using power consumption data.

\section{Conclusions and Future Work}

In this paper, we focus on multi-objective optimal charging control of PHEVs in a multi-level power distribution system. The objectives of enabling valley filling with a higher priority and achieving total cost minimization with a lower priority are both investigated under the system's constraints. For this purpose, a hierarchical optimal algorithm based on the water-filling-based algorithm and the consensus-based method is proposed. Moreover, a moving horizon method is utilized to address the random arrival and departure of PHEVs. Through numerical simulations, we show that our algorithm achieves the desirable performance. In particular, not only is the stability of the power load enhanced, but the global minimization of charging costs for vehicle owners is also achieved by using the proposed algorithm. Further, our algorithm also works for large-scale adoption of PHEVs in the multi-level framework of power distribution system with high adaptability and scalability.

Future work may include non-quadratic cost functions and general communication topologies among the PHEVs. Further, the energy storage function of PHEVs will be taken into account, in which PHEVs are allowed to inject energy back into the power grid, which additionally generates revenue for vehicle owners and enables the peak shaving as distributed generation in the multi-level power distribution system.

Author Contributions: W.L. did theoretical analysis, system implementation, simulations and wrote the first and the last version of the draft paper. The proposed approach was discussed with Z.L. and K.C., who verified the first draft. H.Z. and G.Y. contributed to the literature review.

Funding: This work was supported by the National Key R\&D Program of China (2018YFB0904900, 2018YFB0904904). 
Acknowledgments: This work presents an extended version of a selected paper in the ICCSSE 2017 conference.

Conflicts of Interest: The authors declare no conflict of interest.

\section{References}

1. Iodice, P.; Senatore, A. Industrial and Urban Sources in Campania, Italy: The Air Pollution Emission Inventory. Energy Environ. 2016, 26, 1305-1317. [CrossRef]

2. Iodice, P.; Senatore, A. Experimental-analytical investigation to estimate an emission inventory from road transport sector. In IAENG Transactions on Engineering Sciences-Special Issue of the International MultiConference of Engineers and Computer Scientists, IMECS 2013 and World Congress on Engineering 2013, 1st ed.; Ao, S.I., Chan, A.H.S., Katagiri, H., Xu, L., Eds.; CRC Press: London, UK, 2014; pp. 141-149.

3. Amjad, M.; Ahmad, A.; Rehmani, M.H.; Umer, T. A review of EVs charging: From the perspective of energy optimization, optimization approaches, and charging techniques. Transp. Res. Part D Transp. Environ. 2018, 62, 386-417. [CrossRef]

4. Wijeweera, G.; Annakkage, U.D.; Zhang, W.; Rajapakse, A.D.; Rheault, M. Development of an Equivalent Circuit of a Large Power System for Real-Time Security Assessment. IEEE Trans. Power Syst. 2018, 33, 3490-3499. [CrossRef]

5. Chen, Y.W.; Chang, J.M. Fair Demand Response With Electric Vehicles for the Cloud Based Energy Management Service. IEEE Trans. Smart Grid. 2016, 9, 458-468. [CrossRef]

6. Javaid, N.; Hafeez, G.; Iqbal, S.; Alrajeh, N.; Alabed, M.S.; Guizani, M. Energy Efficient Integration of Renewable Energy Sources in the Smart Grid for Demand Side Management. IEEE Access. 2018, 6, 77077-77096. [CrossRef]

7. Shao, S.; Pipattanasomporn, M.; Rahman, S. Demand response as a load shaping tool in an intelligent grid with electric vehicles. IEEE Trans. Smart Grid. 2011, 2, 624-631. [CrossRef]

8. Ma, Z.; Callaway, D.S.; Hiskens, I.A. Decentralized charging control of large populations of plug-in electric vehicles. IEEE Trans. Control Syst. Technol. 2013, 21, 67-78. [CrossRef]

9. Gan, L.; Topcu, U.; Low, S.H. Optimal decentralized protocol for electric vehicle charging. IEEE Trans. Power Syst. 2013, 28, 940-951. [CrossRef]

10. Tushar, W.; Saad, W.; Poor, H.V.; Smith, D.B. Economics of electric vehicle charging: A game theoretic approach. IEEE Trans. Smart Grid. 2012, 3, 1767-1778. [CrossRef]

11. Rotering, N.; Ilic, M. Optimal charge control of plug-in hybrid electric vehicles in deregulated electricity markets. IEEE Trans. Power Syst. 2011, 26, 1021-1029. [CrossRef]

12. Kang, Q.; Feng, S.W.; Zhou, M.C.; Ammari, A.C.; Sedraoui, K. Optimal Load Scheduling of Plug-In Hybrid Electric Vehicles via Weight-Aggregation Multi-Objective Evolutionary Algorithms. IEEE Trans. Intell. Transp. Syst. 2017, 18, 2557-2568. [CrossRef]

13. Zakariazadeh, A.; Jadid, S.; Siano, P. Multi-objective scheduling of electric vehicles in smart distribution system. Energy Convers. Manag. 2014, 79, 43-53. [CrossRef]

14. Yu, R.; Ding, J.; Zhong, W.; Liu, Y.; Xie, S. PHEV charging and discharging cooperation in V2G networks: A coalition game approach. IEEE Internet Things J. 2014, 1, 578-589. [CrossRef]

15. Wang, T.; Zheng, Z.; Rehmani, M.H.; Yao, S.; Huo, Z. Privacy preservation in big data from the communication perspective-A survey. IEEE Commun. Surv. Tutorials. 2019, 21, 753-778. [CrossRef]

16. Mohsenian-Rad, A.H.; Wong, V.W.S.; Jatskevich, J.; Schober, R.; Leon-Garcia, A. Autonomous demand-side management based on game-theoretic energy consumption scheduling for the future smart grid. IEEE Trans. Smart Grid. 2010, 1, 320-331. [CrossRef]

17. Kazemi, M.; Zareipour, H. Long-term scheduling of battery storage systems in energy and regulation markets considering battery's lifespan. IEEE Trans. Smart Grid. 2018, 9, 6840-6849. [CrossRef]

18. Mou, Y.; Xing, H.; Lin, Z.; Fu, M. A new approach to distributed charging control for plug-in hybrid electric vehicles. In Proceedings of the Proceedings of the 33rd Chinese Control Conference (CCC), Nanjing, China, 28-30 July 2014; pp. 8118-8123.

19. Li, W.; Lin, Z.; Xu, Y.; Zhang, J.; Song, S.; Wang, Y.; Yan, G. An expanded distributed algorithm for dynamic resource allocation over strongly connected topologies. In Proceedings of the 2017 3rd IEEE International Conference on Control Science and Systems Engineering (ICCSSE), Beijing, China, 17-19 August 2017; pp. 500-505. 
20. Xing, H.; Fu, M.; Lin, Z.; Mou, Y. Decentralized optimal scheduling for charging and discharging of plug-in electric vehicles in smart grids. IEEE Trans. Power Syst. 2016, 31, 4118-4127. [CrossRef]

21. Mou, Y.; Xing, H.; Lin, Z.; Fu, M. Decentralized optimal demand-side management for phev charging in a smart grid. IEEE Trans. Smart Grid. 2015, 6, 726-736. [CrossRef]

22. LEAF Electric Car Charging. Available online: http://www.nissanusa.com/electric-cars/leaf/charging-range/ charging (accessed on 1 January 2014).

23. Features and Specs. Available online: http://www.teslamotors.com/models/features\#/battery (accessed on 1 January 2014).

(C) 2019 by the authors. Licensee MDPI, Basel, Switzerland. This article is an open access article distributed under the terms and conditions of the Creative Commons Attribution (CC BY) license (http://creativecommons.org/licenses/by/4.0/). 